\title{
EXTREME FUNCTIONALS ON AN UPPER SEMICONTINUOUS FUNCTION SPACE
}

\author{
F. CUNNINGHAM, JR. AND NINA M. ROY
}

\begin{abstract}
A representation theorem is given for the extreme points of the dual ball of a vector valued function space $X$ with upper semicontinuous norm defined on a compact Hausdorff space $\Omega$. This generalizes the Arens-Kelley theorem which is the case $X=C(\Omega)$.
\end{abstract}

One of the most powerful tools in the abstract geometric description of Banach spaces $X$ which are given by concrete representations is knowing explicitly what the extreme points of the unit ball in $X^{*}$ are. The prototype theorem of this type is the theorem of Arens-Kelley to the effect that if $X=C(\Omega), \Omega$ compact Hausdorff, the extreme points of its dual ball are exactly the evaluations at points of $\Omega$ up to multiplication by scalars of absolute value 1 . The purpose of this note is to generalize this result in two directions: (1) replace continuity of the functions by upper semicontinuity of their absolute values, and (2) allow the functions to be vector valued. See Theorem I below. The significance of these particular hypotheses is to be found in [1]. Briefly, they make the theorem applicable to arbitrary Banach spaces, viewed from the uniform norm point of view. One of us (Roy) will exploit this generality elsewhere along the lines already indicated. Our second theorem is an application of Theorem 1 to quotient modules to show how our method can be used to obtain a related result announced by W. J. Ströbele [3].

Let $\Omega$ be a compact Hausdorff space, and for each $t \in \Omega$ let $X_{t}$ be a normed linear space. We are interested in linear spaces $X$ of functions $x$ defined on $\Omega$ with $x(t) \in X_{t}$ for each $t$, and satisfying at least the first, and usually both of the following:

(i) (Upper semicontinuity) For each $x \in X$ the norm function $t \mapsto$ $\|x(t)\|$ (norm in $X_{t}$ ) is upper semicontinuous.

(ii) (Module property) For $x \in X$ and $f \in C(\Omega)$ the function $f x$ defined by multiplication $(f x(t)=f(t) x(t)$ for all $t)$ belongs to $X$.

Presented to the Society, September 1, 1972; received by the editors September 18, 1972 and, in revised form, April 23, 1973.

AMS (MOS) subject classifications (1970). Primary 46E40, 46B99; Secondary 54C35.

Key words and phrases. Extreme functional, uniform norm, function space, upper semicontinuous norm.

(c) American Mathematical Society 1974 
Of course (i) makes the functions in $X$ bounded, and $X$ becomes a normed space with the uniform norm: $\|x\|=\sup _{t}\|x(t)\|$. For convenience we assume that $X_{t}=\{x(t): x \in X\}$ for each $t$. Denote by $B$ the unit ball in $X^{*}$ and by $E$ the set of extreme points of $B$. Analogous notations apply with the subscript $t$ throughout. For $t \in \Omega$ and $q \in X_{t}^{*}$ define the "evaluation functional" $e_{t} q \in X^{*}$ by $e_{t} q(x)=q(x(t))$. Then $e_{t}: X_{t}^{*} \rightarrow X^{*}$ is an injective linear map of norm $\leqq 1$.

THEOREM 1. (a) If $X$ satisfies (i), then

$$
E \subset \bigcup\left\{e_{t}\left(E_{t}\right): t \in \Omega\right\} .
$$

(b) If $X$ satisfies (i) and (ii), then

$$
E=\bigcup\left\{e_{t}\left(E_{t}\right): t \in \Omega, X_{t} \neq\{0\}\right\} \text {. }
$$

The proof generalizes a proof of the Arens-Kelley theorem as given, for example, in [2, p. 441]. Let $Q=\bigcup\left\{e_{t}\left(B_{t}\right): t \in \Omega\right\}$.

LEMMA 1. $Q$ is weak* closed.

Proof. Suppose $p \in X^{*}$ is the weak* limit of a net $\left\{p_{\alpha}\right\}=\left\{e_{t_{\alpha}} q_{\alpha}\right\}$ in $Q$, where $t_{\alpha} \in \Omega, q_{\alpha} \in B_{t_{\alpha}}$. Since $\Omega$ is compact we may assume that $t_{\alpha}$ converges to some $t \in \Omega$. Then for any $x \in X$ we obtain from (i) the inequality

$$
\begin{aligned}
|p(x)| & =\lim \left|p_{\alpha}(x)\right|=\lim \left|q_{\alpha}\left(x\left(t_{\alpha}\right)\right)\right| \\
& \leqq \lim \sup \left\|q_{\alpha}\right\| \cdot\left\|x\left(t_{\alpha}\right)\right\| \leqq\|x(t)\| .
\end{aligned}
$$

In particular if $x(t)=0$ it follows that $p(x)=0$. Thus a linear functional $q$ is well defined on $X_{t}$ by $q(x(t))=p(x)$. The above inequality then shows that $q$ is bounded, in fact that $q \in B_{t}$. Since clearly $p=e_{t} q$, we have $p \in Q$ and the lemma is proved.

LEMMA 2. $\mathrm{cl} \operatorname{co} Q=B$.

Proof. We mean on the left the weak* closed convex hull of $Q$. Since $Q \subset B$ and $B$ is convex and weak* compact, the inclusion cl co $Q \subset B$ is clear. Conversely, observe that the polar of $Q$ in $X$ is contained in the unit ball, that is, $Q^{\circ} \subset B^{\circ}$. Also, since cl co $Q$ is balanced (because $Q$ is), we have $Q^{\circ \circ}=\mathrm{cl} \operatorname{co} Q[4$, Theorem 1, p. 238]. Therefore $\operatorname{cl} \operatorname{co} Q=$ $Q^{\circ \circ} \supset B^{\circ \circ}=B$.

Proof of Theorem 1 (a). According to Lemmas 1 and 2, [2, Lemma 5, p. 440] applies and shows that $E \subset Q$. Thus any $p \in E$ is of the form $p=e_{t} q$ for some $t \in \Omega, q \in B_{t}$. But then $q$ must be an extreme point of $B_{t}$ because $p$ is an extreme point of $B$ and $e_{t}: B_{t} \rightarrow B$ is linear and injective.

For part (b) of the theorem, define the set of functionals with support 
$\{t\}(t \in \Omega)$ as follows:

$$
A_{t}=\left\{p \in X^{*}: x \in X \text { and } x(t)=0 \text { imply } p(x)=0\right\} .
$$

Lemma 3. $e_{t}$ is an isometry of $X_{t}^{*}$ onto $A_{t}$.

Proof. Clearly $e_{t}$ maps $X_{t}^{*}$ into $A_{t}$. We construct its inverse $a_{t}$ as follows. For $p \in A_{t}$, a linear functional $a_{t} p$ on $X_{t}$ is well defined by $a_{t} p(x(t))=p(x)$ for $x \in X$. To show that $a_{t} p$ is bounded and to estimate its norm, let $\varepsilon>0$ be given. For an element $x \in X$ such that $x(t) \neq 0$, by the upper semicontinuity of the norm there is a neighborhood $U$ of $t$ such that $\|x(s)\| \leqq(1+\varepsilon)\|x(t)\|$ for $s \in U$. Let $f \in C(\Omega)$ satisfy $|f| \leqq 1, f(t)=1, f=0$ outside $U$. Then $f x \in X,\|f x\| \leqq(1+\varepsilon)\|x(t)\|$ and

$$
\begin{aligned}
\left|a_{t} p(x(t))\right| & =\left|a_{t} p(f x(t))\right|=|p(f x)| \\
& \leqq\|p\| \cdot\|f x\| \leqq(1+\varepsilon)\|p\| \cdot\|x(t)\| .
\end{aligned}
$$

This shows that $a_{t} p \in X_{t}^{*}$ and $a_{t}: A_{t} \rightarrow X_{t}^{*}$ has norm $\leqq 1$. Since $a_{t}$ and $e_{t}$ are clearly inverses of each other, and both are of norm $\leqq 1$, they are isometries.

Proof of Theorem 1 (b). It remains to show $e_{t}\left(E_{t}\right) \subset E$ for $t \in \Omega$, $X_{t} \neq\{0\}$. Suppose to the contrary that $p=e_{t} q$ where $q \in E_{t}, X_{t} \neq\{0\}$, and $p \notin E$. Then $p=\left(p_{1}+p_{2}\right) / 2$ where $p_{1}, p_{2} \in B$ and $p_{1} \neq p_{2}$. If we can prove $p_{1}, p_{2} \in A_{t}$, then by Lemma 3 we shall have a contradiction. To this end, let $x \in X$ with $x(t)=0$, and let $\varepsilon>0$ be given. By the upper semicontinuity of the norm of $x$ there is a neighborhood $U$ of $t$ such that $\|x(s)\|<\varepsilon$ for $s \in U$. Find $f \in C(\Omega)$ satisfying $|f| \leqq 1, f(t)=1$, and $f=0$ outside $U$. Also, from the fact that $\|q\|=1$ find $y \in X$ so that $\|y\| \leqq 1$ and $q(y(t))$ is real and $>1-\varepsilon$. Set $z=f y$. Then $q(z(t))=q(y(t))>1-\varepsilon$ and $\|z\| \leqq 1$. Moreover, since $z$ is 0 outside $U$ and $\|x(s)\| \leqq \varepsilon$ for $s$ in $U$, we have $\|x+z\| \leqq 1+\varepsilon$. Now $\left|p_{1}(z)\right| \leqq\left\|p_{1}\right\| \cdot\|z\| \leqq 1$, and similarly $\left|p_{2}(z)\right| \leqq 1$. Thus $p_{1}(z)$ and $p_{2}(z)$ are complex numbers in the unit disk whose midpoint $p(z)$ is real and $>1-\varepsilon$. Therefore

$$
\left|p_{1}(z)-p_{2}(z)\right|<2 \sqrt{ }\left(1-(1-\varepsilon)^{2}\right)<2 \sqrt{ }(2 \varepsilon) .
$$

The same argument applied to $x+z$ in place of $z$ (with the disk slightly enlarged) gives

$$
\left|p_{1}(x+z)-p_{2}(x+z)\right|<4 \sqrt{ } \varepsilon \text {. }
$$

Combining these gives $\left|p_{1}(x)-p_{2}(x)\right|<8 \sqrt{ } \varepsilon$, whence $p_{1}(x)-p_{2}(x)=0$. Thus $p_{1}-p_{2} \in A_{t}$. Since also $\left(p_{1}+p_{2}\right) / 2=p \in A_{1}$, we have $p_{1}, p_{2} \in A_{t}$ as required, completing the proof of Theorem 1.

In Theorem 2 we assume that $X$ is a function space on $\Omega$ satisfying both (i) and (ii) and that $Y \subset X$ is a subspace, not necessarily closed, which is a $C(\Omega)$-submodule, that is, $Y$ is invariant under the action of 
$C(\Omega)$ by multiplication. To avoid triviality assume $\bar{Y} \neq X$, and let $Y^{\perp}=$ $\left\{p \in X^{*}: p(y)=0\right.$ for all $y$ in $\left.Y\right\}$. Then $X / \bar{Y}$ becomes a normed linear space in the usual way, and $(X \mid \bar{Y})^{*}$ can be identified isometrically with $Y^{\perp}$, the dual unit ball being $Y^{\perp} \cap B$.

For $t \in \Omega$ define $Y_{t}=\{y(t): y \in Y\}$. The above notations are repeated with the subscript $t$, giving an isometry of $\left(X_{t} / \bar{Y}_{t}\right) *$ with $Y_{t}^{\perp}$, the unit ball here being $Y_{t} \cap \cap B_{t}$. For any set $S$ we write $\operatorname{Ext}(S)$ for the set of extreme points of $S$.

TheOREM 2. $\operatorname{Ext}\left(Y^{\perp} \cap B\right)=\bigcup\left\{e_{t}\left(\operatorname{Ext}\left(Y_{t}^{\perp} \cap B_{t}\right)\right): t \in \Omega, \bar{Y}_{t} \neq X_{t}\right\}$.

The proof will be an application of Theorem 1. To make Theorem 1 applicable we need to construe $X / \bar{Y}$ as a function space on $\Omega$ satisfying (i) and (ii) with $X_{t} / \bar{Y}_{t}$ as component space at $t \in \Omega$. The key to this is the following lemma.

Lemma 4. Let $x \in X$. Then

$$
\inf _{y \in Y}\|x-y\|=\sup _{t \in \Omega} \inf _{y \in Y}\|x(t)-y(t)\| .
$$

Proof. Let the right member of this equation be called $d$. Since for $t \in \Omega$ and $y \in Y,\|x(t)-y(t)\|=\|(x-y)(t)\| \leqq\|x-y\|$, we obtain by taking infimum over $y \in Y$ and then supremum over $t \in \Omega$ that $d \leqq \inf _{y \in Y}\|x-y\|$.

For the converse inequality let $\varepsilon>0$ be given, and for each $t \in \Omega$ choose $y_{t} \in Y$ so that $\left\|x(t)-y_{t}(t)\right\|<d+\varepsilon$. Then since $s \mapsto\left\|x(s)-y_{t}(s)\right\|$ is upper semicontinuous we can find for each $t$ an open neighborhood $U_{t}$ of $t$ such that $\left\|x(s)-y_{t}(s)\right\|<d+\varepsilon$ for all $s \in U_{t}$. Let $\left\{U_{1}, \cdots, U_{n}\right\}$ be a finite covering of $\Omega$ by such neighborhoods. Let the corresponding points $t$ be $t_{1}, \cdots, t_{n}$ and let $y_{i}=y_{t_{i}}(i=1, \cdots, n)$. Let $\left\{f_{1}, \cdots, f_{n}\right\}$ be a continuous partition of 1 subordinate to the covering $\left\{U_{1}, \cdots, U_{n}\right\}$ and set $y=$ $\sum_{i=1}^{n} f_{i} y_{i}$. Then $y \in Y$, and for each $t \in \Omega$ we have

$$
\begin{aligned}
\|x(t)-y(t)\| & =\left\|\sum f_{i}(t)\left[x(t)-y_{i}(t)\right]\right\| \\
& \leqq \sum_{t \in U_{i}} f_{i}(t)\left\|x(t)-y_{i}(t)\right\|<d+\varepsilon .
\end{aligned}
$$

Taking supremum over $t \in \Omega$ gives $\|x-y\| \leqq d+\varepsilon$, and, $\varepsilon$ being arbitrary, the lemma is proved.

Proof of Theorem 2. For each $x \in X$ define a function $\tilde{x}$ on $\Omega$ by $\tilde{x}(t)=x(t)+\bar{Y}_{t}$ (an element of $\left.X_{t} / \bar{Y}_{t}\right)$. Let $\tilde{X}$ be the function space $\{\tilde{x}: x \in X\}$ with the uniform norm: $\|\tilde{x}\|=\sup _{t}\|\tilde{x}(t)\|=d$ of Lemma 4. According to Lemma 4 (with $d=0$ ) $\tilde{x}(t)=0$ if and only if $x \in \bar{Y}$, so that $x+\bar{Y} \leftrightarrow \tilde{X}$ is an isomorphism of $X / \bar{Y}$ with $\tilde{X}$. Lemma 4 states that this isomorphism is in fact an isometry. With the identifications $(X / \bar{Y})^{*}=Y^{\perp}$ and $\left(X_{t} / \bar{Y}_{t}\right)^{*}=Y_{t}^{-}$, Theorem 2 follows from Theorem 1 as soon as we 
show that $\tilde{X}$ satisfies (i) and (ii). To verify (i) note that for $x \in X$

$$
\|\tilde{x}(t)\|=\inf _{y \in Y}\|x(t)-y(t)\| .
$$

Since for each $y \in Y, t \mapsto\|x(t)-y(t)\|$ is upper semicontinuous, it follows that $t \mapsto\|\tilde{x}(t)\|$ is also. Finally, (ii) is immediate from the identity $(f x)^{\sim}(t)=$ $f(t) \tilde{x}(t)$ which holds for $f \in C(\Omega), x \in X, t \in \Omega$. Theorem 2 is proved.

The following special case of Theorem 2 was announced (in part) by W. J. Ströbele [3]. Let $T$ be locally compact Hausdorff, $S \subset T$ a nonempty subset. Let $N$ be a normed linear space, and $M \subset N$ a linear subspace, not necessarily closed. Let $X=C_{0}(T, N)$ be the normed space (with the uniform norm) of all continuous functions on $T$ to $N$ which vanish at infinity, and let $Y=\{x \in X: x(S) \subset M\}$. For $q \in N^{*}$ and $t \in T$ define $e_{t} q \in X^{*}$ by $e_{t} q(x)=q(x(t))$. Finally, write $B_{N}$ and $B_{X}$ for the unit balls in $N^{*}$ and $X^{*}$ respectively.

Corollary. $\operatorname{Ext}\left(Y^{\perp} \cap B_{X}\right)=\bigcup\left\{e_{t}\left(\operatorname{Ext}\left(M^{\perp} \cap B_{N}\right)\right): t \in \bar{S}\right\}$.

Proof. Let $\Omega=T \cup\{\infty\}$ be the one-point compactification of $T$. Extend the functions in $X$ to $\Omega$ by always setting $x(\infty)=0$. The conditions of Theorem 2 are evidently satisfied by the pair $X, Y$. Here $M \subset Y_{t} \subset \bar{M}$ for $t \in \bar{S}$, whereas $Y_{t}=N$ for $t \notin S(t \neq \infty)$.

\section{REFERENCES}

1. F. Cunningham, Jr., M-structure in Banach spaces, Proc. Cambridge Philos. Soc. 63 (1967), 613-629. MR 35 \#3415.

2. N. Dunford and J. T. Schwartz, Linear operators. I: General theory, Pure and Appl. Math., vol. 7, Interscience, New York, 1958. MR 22 \#8302.

3. W. J. Ströbele, On the representation of the extremal functionals on $C_{0}(T, X)$, Notices Amer. Math. Soc. 19 (1972), A-443. Abstract 72T-B119.

4. A. Wilansky, Functional analysis, Blaisdell, New York, 1964. MR 30 \#425.

Department of Mathematics, Bryn Mawr College, Bryn Mawr, Pennsylvania 19010

Department of Mathematics, Rosemont College, Rosemont, Pennsylvania 19010 